\title{
Los derechos humanos en las estrategias de erradicación de la pobreza: agenda 2030
}

Diana M. Verdiales López

\section{Resumen}

La pobreza constituye una problemática que afecta a toda la comunidad internacional y, por lo tanto, su erradicación se convierte en un asunto de todos. Hasta el momento, no se dispone de un marco jurídico que imponga las obligaciones necesarias para su erradicación, por lo que los Estados tan solo han acordado marcos de actuación que no van más allá de meros compromisos políticos. No obstante, en el 2000 se adoptaron los Objetivos de Desarrollo del Milenio (ODM) y posteriormente en el 2015, los Objetivos de Desarrollo Sostenible (ODS) que se incorporan en la Agenda 2030. Estos instrumentos propenden al establecimiento de Objetivos y Metas para crear una sociedad internacional justa, solidaria, equitativa y sostenible. El Objetivo 1 de dichos instrumentos es la erradicación de la pobreza, por lo que los principios que sustentan este Objetivo está directamente vinculados con el respeto de los derechos humanos, puesto que la pobreza va más allá de un asunto puramente económico y su erradicación requiere indispensablemente del reconocimiento de derechos, sobre todo de las personas más vulnerables. El presente trabajo pretende resaltar, por ello, la importancia que tiene la vinculación que debe existir entre los derechos humanos y la pobreza como estrategia necesaria para su erradicación y el logro de los objetivos plasmados en la Agenda 2030.

Palabras clave: pobreza - derechos humanos, Objetivos de Desarrollo del Milenio - Objetivos de Desarrollo Sostenible - Derecho Internacional

\section{Human rights in poverty eradication strategies: 2030 agenda}

\begin{abstract}
Poverty is a problem that affects the entire international community and, therefore, its eradication becomes a matter for all. So far, there is no legal framework in place to impose the obligations necessary for their eradication, so States have only agreed on frameworks of action that do not go beyond mere political commitments. However, the Millennium Development Goals (MDGs) were adopted in 2000 and subsequently in 2015, the Sustainable Development Goals (SDGs) were incorporated into the 2030 Agenda. These instruments propose the establishment of Goals and Goals to create a just, supportive, equitable and sustainable international society. Objective 1 of these instruments is the eradication of poverty, so the principles underpinning this Objective are directly linked to respect for human rights, since poverty goes beyond a purely economic issue and its eradication essentially require the recognition of rights, especially of the most vulnerable. This work aims to highlight, therefore, the importance of the link between human rights and poverty as a necessary strategy for its eradication and the achievement of the objectives set out in the 2030 Agenda.
\end{abstract}

Key words: poverty - human rights - Millennium Development Goals - Sustainable Development Goals International Law

TRABAJO RECIBIDO: 02/10/2019 TRABAJO ACEPTADO: 29/10/2019

\footnotetext{
* Doctora en Derechos Humanos, Paz y Desarrollo Sostenible por la Universidad de Valencia (España) y Responsable de Proyectos de Investigación y Cooperación al Desarrollo en el Centro de Estudios de Iberoamérica (CEIB) de la Universidad Rey Juan Carlos de Madrid (España). Email: diana.verdiales@urjc.es
} 


\section{Introducción}

El concepto de pobreza ha tenido tantas definiciones como estrategias se han formulado para su combate y erradicación. Interesa adoptar como base, no obstante, la definición que proporciona el Comité de Derechos Económicos, Sociales y Culturales a pesar de que enfatice en que no hay una sola definición de la pobreza al tratarse de un fenómeno multidimensional, por lo que todas pueden ser válidas. En cualquier caso, la definición de pobreza que adopta este Comité sería la siguiente: "la pobreza es una condición humana que se caracteriza por la privación continua o crónica de los recursos, la capacidad, las opciones, la seguridad y el poder necesarios para disfrutar de un nivel de vida adecuado y de otros derechos civiles, culturales, económicos, políticos y sociales" (Alto Comisionado de Naciones Unidas para los Derechos Humanos, 2012:2). En verdad, una definición amplia que cubre bastantes supuestos y que puede resultar útil a la hora de afrontar este fenómeno en la comunidad internacional, puesto que contar con una definición de pobreza así permite singularizar las estrategias que estén orientadas a combatirla.

Ahora bien, el estudio de la pobreza, y sobre todo de las estrategias para combatirla, ha ido cobrando cada vez mayor importancia debido a varios factores: 1) por el mero reconocimiento de que para conseguir el desarrollo humano sostenible para todos los pueblos es imprescindible la erradicación de la pobreza, como estrategia contenida también en los Objetivos de Desarrollo Sostenible (ODS); 2) por solidaridad con los más de 736 millones de personas que actualmente viven en situación de pobreza en todo el mundo (con menos de 1.90 dólares diarios) y; 3 ) porque, según el economista australiano Martin Ravallion, es la primera vez en la historia de la humanidad en la que es posible erradicar la pobreza en el mundo con los ingresos generados por los países desarrollados. En este sentido, las estrategias diseñadas para combatir y erradicar la pobreza han sido numerosas y se han venido desarrollando en el plano internacional, desde hace más de seis decenios. Siempre cabe recordar que ya en la Carta de las Naciones Unidas, en su artículo 55, se afirma la importancia de crear condiciones de estabilidad y bienestar para el establecimiento de relaciones pacíficas y amistosas basadas en los principios de igualdad de derechos y de la libre determinación de los pueblos, promoviendo un nivel de vida más elevado mediante trabajos permanentes para todos y condiciones de progreso y desarrollo económico y social, sin hacer distinción por motivos de raza, sexo, idioma o religión.

En realidad, Naciones Unidas ha liderado la implementación de estrategias de erradicación de la pobreza a nivel mundial, mediante la elaboración de programas específicos llevados a cabo por distintas agencias de esta Organización desde 1960, periodo en el que instauraron mecanismos de fortalecimiento de los derechos humanos y promoción de la asistencia técnica en ayuda humanitaria y en la cooperación internacional. Estrategias de este tipo encontraron, no obstante, un gran impulso a partir de los años noventa, periodo en el que la Organización inició un cambio interno de sus estrategias de promoción de los derechos humanos y erradicación de la pobreza. Es por ello que, en 1992, la Asamblea General de las Naciones Unidas declaró el 17 de octubre como el Día Internacional de la Erradicación de la Pobreza para poner de manifiesto la importancia de este propósito en la agencia internacional. En resumen, el combate contra la pobreza es liderado básicamente por Naciones Unidas y desde una perspectiva de derechos humanos, puesto que, como lo indica la propia Organización, "la pobreza es en sí misma un problema de derechos humanos urgente y es a la vez causa y consecuencia de violaciones de los derechos humanos, pues se caracteriza por vulneraciones múltiples e interconexas de los derechos civiles, políticos, económicos, sociales y culturales, y las personas que viven en ella se ven expuestas regularmente a la denegación de su dignidad e igualdad"2.

\footnotetext{
${ }^{2}$ El día Internacional para la Erradicación de la Pobreza, establecido por Naciones Unidas es el 17 de octubre. Mayor información ver en: https://www.un.org/es/events/povertyday/
} 
Posteriormente, dada la importancia de seguir trabajando en el combate contra la pobreza, cabe destacar la celebración de la Cumbre Mundial sobre Desarrollo Social en 1995, en Copenhague, que se centra también en tres problemáticas mundiales identificadas: la erradicación de la pobreza, la generación de empleo y la integración social para crear una comunidad internacional que permita la construcción de sociedades seguras, justas, libres y armoniosas que generen oportunidades y mejores las condiciones de vida ${ }^{3}$. Más aún, en diciembre de ese mismo año, la Asamblea General de Naciones Unidas inaugura el primer Decenio de Naciones Unidas para la erradicación de la pobreza $(1997-2006)^{4}$, fijando un año después el tema que definiría el siguiente decenio en las estrategias de erradicación de la pobreza: "pleno empleo y trabajo decente para todos" (A/RES/63/230, 2009:2) en el que la erradicación de la pobreza debe ser concebida como un imperativo ético, social, político y económico de la humanidad.

Pero, también, otras instituciones se han sumado a la lucha y combate contra la pobreza como es el caso, entre otras, de la Organización Internacional del Trabajo (OIT), la Organización Mundial de la Salud, el Grupo Banco Mundial, o el Fondo Monetario Internacional. En todos los casos, la mayoría de estas Organizaciones, así como el resto de las agencias de Naciones Unidas, ponen de relieve que el desarrollo mundial sólo será posible cuando se invierta en las personas y en las instituciones. Por ello precisamente, se crearon una serie de Objetivos globales para trabajar por un desarrollo integral de las personas, que quedaron plasmados en la Cumbre del Milenio llevada a cabo en el año 2000 en Nueva York ${ }^{5}$. Cabe recordar que esta Cumbre ponía de manifiesto la necesidad de seguir trabajando por un mundo más equitativo, justo y solidario que generara el desarrollo y bienestar integral que todo ser humano requería para llevar una vida digna. Así, se formularon 8 Objetivos a implantar en los países en desarrollo por un periodo de 15 años y, en particular, como indica la Declaración del Milenio, se trata de "(...) crear en los planos nacional y mundial un entorno propicio al desarrollo y a la eliminación de la pobreza" (A/RES/55/2, 2000).

No obstante, en 2015, se adopta la Resolución 70/1 por la Asamblea General de las Naciones Unidas, titulada "Transformar nuestro mundo: la Agenda 2030 para el Desarrollo Sostenible" en la que se incorporan 17 Objetivos y 169 metas. Esta vez se considera necesaria su implementación no sólo en los países menos desarrollados sino también en los países desarrollados para evitar disparidades económicas y sociales a nivel internacional. En esta Resolución se parte de la afirmación de que "la erradicación de la pobreza en todas sus formas y dimensiones, incluida la pobreza extrema, es el mayor desafío a que se enfrenta el mundo y constituye un requisito indispensable para el desarrollo sostenible" (A/RES/70/1, 2015:1) y, verdaderamente, la elaboración de esta Agenda ha constituido un hecho bastante transcendental en la lucha contra este fenómeno.

Lo importante a destacar, en todo caso, es que en ambos instrumentos la erradicación de la pobreza se concibe precisamente como el primer Objetivo a lograr por la comunidad internacional y, por ende, debe entenderse como un propósito prioritario en el cumplimiento de los Objetivos de Desarrollo. Así lo ha concebido la OIT al decir que "la Agenda 2030 es el instrumento con que la comunidad internacional se ha dotado para culminar con éxito uno de los cometidos centrales que se encomendaron a la OIT en el momento de su fundación: convertir a la pobreza en un recuerdo del pasado" (OIT, 2016:1). Más todavía, para lograrlo se estima

\footnotetext{
3 Véanse los resultados en https://www.un.org/development/desa/dspd/world-summit-for-socialdevelopment-1995.html

${ }^{4}$ El coordinador responsable del Decenio de las Naciones Unidas para la Erradicación de la Pobreza es la División de Política Social y Desarrollo Social (DSPD) del Departamento de Asuntos Económicos y Sociales (DAES). Esta División realiza actividades que facilitan y ayudan a los gobiernos con la aplicación efectiva de estrategias para dar cumplimiento a los compromisos adquiridos en materia de desarrollo social.

${ }^{5}$ Se pueden ver los documentos más relevantes en https://www.un.org/spanish/milenio/
} 
preciso que la pobreza quede vinculada a la protección de los derechos humanos por ser éste el camino más eficaz para alcanzar su erradicación. En resumen, se establece así una estrecha y profunda vinculación entre pobreza y respeto de los derechos humanos.

\section{Principales fundamentos de la lucha contra la pobreza en la agenda global}

A pesar de no siempre se haga expresamente, tanto los ODM como los ODS incorporan principios que sustentan el respeto de los derechos humanos emanados de la Declaración Universal de los Derechos Humanos, así como de la Carta de las Naciones Unidas y de otros instrumentos internacionales. En este sentido, cabría afirmar que los Objetivos de Desarrollo han tenido y tienen entre sus principales propósitos trabajar conjuntamente en el combate y erradicación de la pobreza incorporando los derechos humanos en su desarrollo e implementación para que "nadie se quede atrás" (A/RES/70/1, 2015:13 y 36).

\subsection{Objetivo de Desarrollo del Milenio (ODM 1). Erradicar la pobreza extrema y el hambre. Resultados reales $v s$ resultados esperados}

El primer objetivo que plasma la Declaración del Milenio fue la erradicación de la pobreza y el hambre por lo que ésta es, con toda seguridad, la mayor preocupación de los gobiernos del mundo en el ámbito del desarrollo. En ese sentido, los ODM plasmaron las siguientes metas para su implementación en los países que adoptaron esta Declaración:

Meta 1.A: Reducir a la mitad, entre 1990 y 2015, la proporción de personas con ingresos inferiores a 1,25 dólares al día. El objetivo de reducir a la mitad las tasas de pobreza extrema se alcanzó cinco años antes de la fecha límite fijada para 2015. La tasa mundial de pobreza de personas que vivían con menos de 1.25 dólares al día descendió en 2010 a menos de la mitad de la registrada en 1990. La cantidad de personas que vivían en pobreza extrema se redujo en más de la mitad, cayendo de 1.900 millones en 1990 a 836 millones en 2015. Sin embargo, todavía se encontraban más de 800 millones de personas en situación de pobreza. Asimismo, se calculaba que en 2015 más de 16.000 niños menores de 5 años morían diariamente por motivos prevenibles, siendo la tasa de mortalidad materna 14 veces mayor en las regiones en desarrollo que en las regiones desarrolladas (ONU, 2015:9).

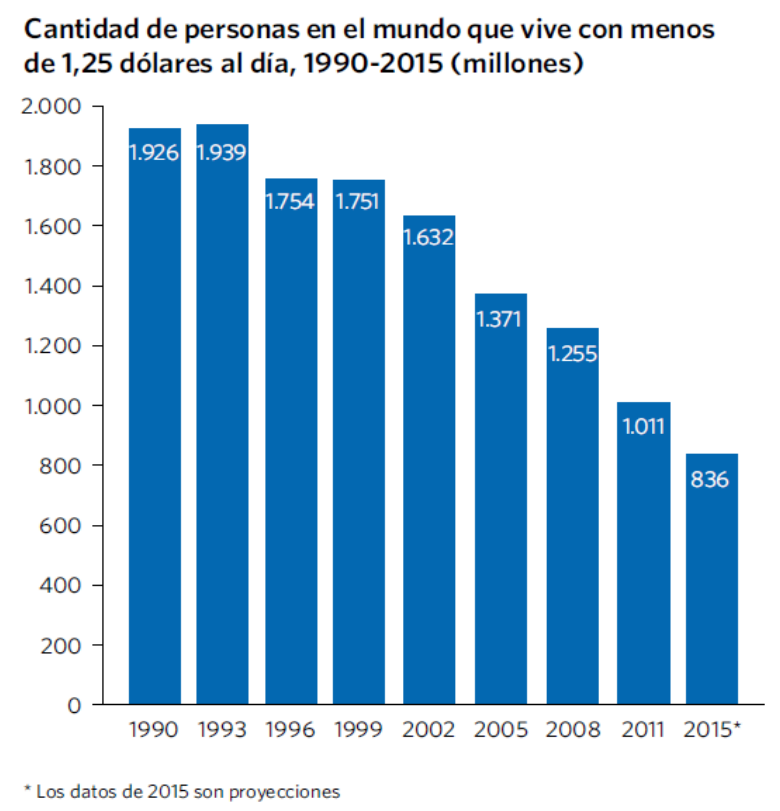

Fuente: NACIONES UNIDAS (2015). “Objetivos de Desarrollo del Milenio. Informe de 2015”, Nueva York, p. 9 
Meta1.B: Alcanzar el empleo pleno y productivo y un trabajo decente para todos, incluidos las mujeres y los jóvenes. En el 2000 el $32.7 \%$ de la población ocupada vivía con ingresos inferiores a 1.25 dólares, mientras que para el 2014 el porcentaje se redujo al $11.8 \%$. En 2011, 384 millones de trabajadores en el mundo vivían por debajo del umbral de pobreza con 1,25 dólares al día, lo que supone una reducción de 294 millones desde 2001. Sin embargo, según datos de la OIT, más de 204 millones de personas estaban desempleadas en 2015, representando a 34 millones más antes del inicio de la crisis mundial del 2008 y 53 millones más que en 1991. Durante este periodo de crisis económica las oportunidades de empleo disminuyeron tanto en las regiones en desarrollo como en las desarrolladas, cayendo $3.3 \%$ de 1991 a 2015 en las regiones en desarrollo y un 1\% en las regiones desarrolladas durante ese mismo periodo ${ }^{6}$. En particular, persiste la desigualdad de género en la tasa de empleo, que en 2012 alcanzaba una diferencia de $24.8 \%$ entre hombres y mujeres.

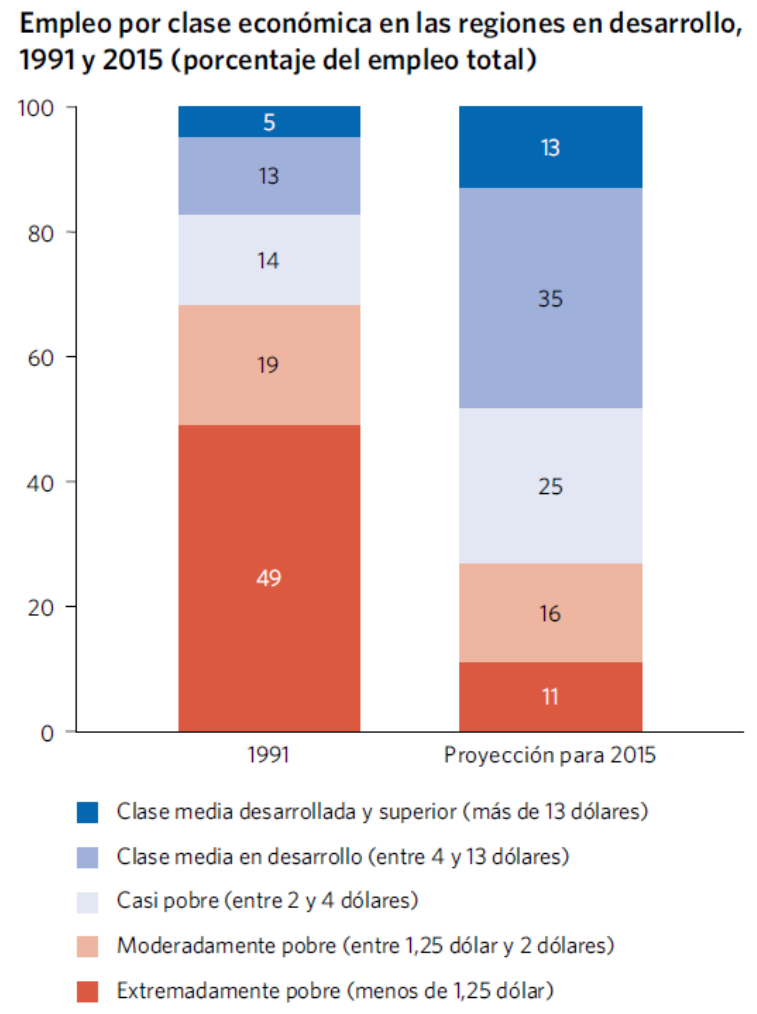

Fuente: NACIONES UNIDAS (2015). “Objetivos de Desarrollo del Milenio. Informe de 2015”, Nueva York, p. 18

Meta 1.C: Reducir a la mitad, entre 1990 y 2015, la proporción de personas que padecen hambre. Pese a que se registró una reducción significativa en la proporción de personas con desnutrición, no se logró el objetivo de reducir a la mitad dicha cifra a nivel mundial, ya que en ello influyeron numerosos factores tales como el aumento del desempleo, los altos y volátiles precios de los alimentos, los incidentes meteorológicos y los desastres naturales, entre otros. Se calcula que en todo el mundo hay 842 millones de personas desnutridas. Todavía más de 99 millones de niños menores de cinco años están desnutridos y tienen un peso inferior al normal. El porcentaje de la población mundial que no alcanzaba el

\footnotetext{
${ }^{6}$ Siendo las reducciones más importantes en Asia Oriental y Meridional, donde se registraron caídas en el empleo de más de $6.7 \%$ y $4.6 \%$, respectivamente. Véase en: NACIONES UNIDAS (2015). "Objetivos de Desarrollo del Milenio. Informe de 2015”, Nueva York, p. 17
} 
nivel mínimo de consumo de energía alimentaria en el 2000 era del 14.9 y para el 2015 fue del 10.9\% (Naciones Unidas 2015:20).

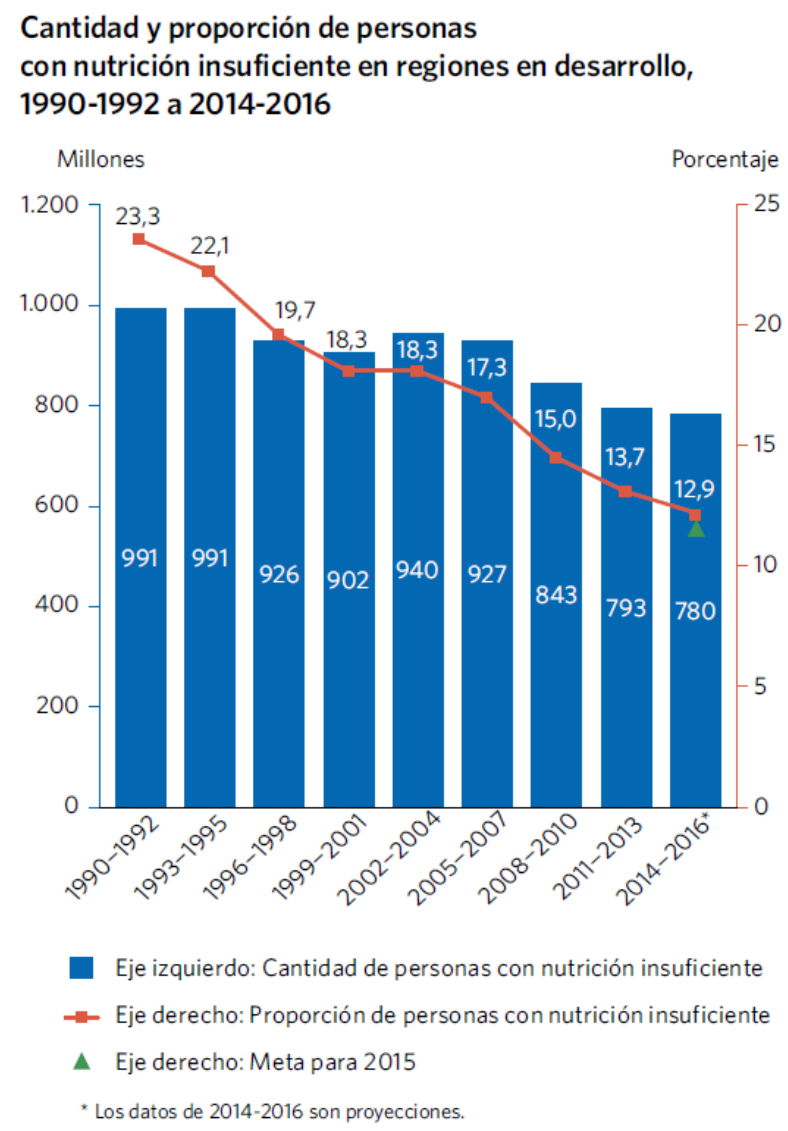

Fuente: NACIONES UNIDAS (2015) “Objetivos de Desarrollo del Milenio. Informe de 2015”, Nueva York, p. 20

\subsection{Objetivo de Desarrollo Sostenible 1. Fin de la pobreza. Alcances y retos}

La necesidad de seguir trabajando en los aspectos no conseguidos en los ODM dieron como resultado la elaboración de nuevas propuestas que culminaron en la iniciativa de la Agenda 2030 más amplia e incluyente. Prueba de ello es que se puede advertir que, a diferencia de los ODM, los ODS pretenden ir más allá del combate contra el hambre y la pobreza, hacia una visión más global e integral en el ámbito del desarrollo humano sostenible. Esto explica que no solo haya aumentado el número de Objetivos y Metas sino que, también, se incrementaron las áreas de acción incluyendo los aspectos sociales, económicos y medioambientales. En cualquier caso, el primer Objetivo de esta nueva Agenda también estaba dedicado a la erradicación de la pobreza, siendo de igual o mayor importancia que en los Objetivos anteriores. En concreto el ODS 1 se dividió en 5 metas (A/RES/70/1, 2015:17):

"1.1 Para 2030, erradicar la pobreza extrema para todas las personas en el mundo, actualmente medida por un ingreso por persona inferior a 1.25 dólares de los Estados Unidos al día.

1.2 Para 2030, reducir al menos a la mitad la proporción de hombres, mujeres y niños de todas las edades que viven en la pobreza en todas sus dimensiones con arreglo a las definiciones nacionales.

1.3 Poner en práctica a nivel nacional sistemas y medidas apropiadas de protección social para todos, incluidos niveles mínimos y, para 2030, lograr una amplia cobertura de los pobres y los vulnerables. 
1.4 Para 2030, garantizar que todos los hombres y mujeres, en particular los pobres y los vulnerables, tengan los mismos derechos a los recursos económicos, así como acceso a los servicios básicos, la propiedad y el control de las tierras y otros bienes, la herencia, los recursos naturales, las nuevas tecnologías apropiadas y los servicios financieros, incluida la microfinanciación.

1.5 Para 2030, fomentar la resiliencia de los pobres y las personas que se encuentran en situaciones vulnerables y reducir su exposición y vulnerabilidad a los fenómenos extremos relacionados con el clima y otras crisis y desastres económicos, sociales y ambientales

1.a. Garantizar una movilización importante de recursos procedentes de diversas fuentes, incluso mediante la mejora de la cooperación para el desarrollo, a fin de proporcionar medios suficientes y previsibles a los países en desarrollo, en particular los países menos adelantados, para poner en práctica programas y políticas encaminados a poner fin a la pobreza en todas sus dimensiones

1.b. Crear marcos normativos sólidos en los planos nacional, regional e internacional, sobre la base de estrategias de desarrollo en favor de los pobres que tengan en cuenta las cuestiones de género, a fin de apoyar la inversión acelerada en medidas para erradicar la pobreza".

Aunque es todavía prematuro hacer una valoración sobre los resultados obtenidos en relación con estas metas, cabría decir que: unos 783 millones de personas viven por debajo del umbral de pobreza, con 1.90 dólares diarios; que en 2016, menos del 10\% de los trabajadores de todo el mundo vivían con sus familias con menos de 1.90 dólares diarios por persona; que en el mundo existen 122 mujeres, entre los 25 y 34 años, viviendo en extrema pobreza por cada 100 hombres del mismo grupo de edades; que uno de cada cuatro niños menores de cinco años, en todo el mundo, tiene una estatura inadecuada para su edad; que solo el $45 \%$ de la población mundial estaba amparada efectivamente por un sistema de protección social con al menos una prestación en efectivo en 2016; y que la mayoría de las personas que se encuentran por debajo del umbral de pobreza viven en dos regiones: Asia meridional y África subsahariana ${ }^{7}$. Los datos, desde luego, resultan desalentadores pero, sobre todo, permiten comprobar que todavía la comunidad internacional debe hacer un ingente esfuerzo en la lucha contra la pobreza.

\section{Estrategias políticas, sociales y medioambientales para la erradicación de la pobreza en los ODS}

Como hemos hecho mención, la pobreza tiene muchas dimensiones y, además, sus principales causas son el desempleo, la exclusión social y la alta vulnerabilidad de determinadas poblaciones a los desastres naturales, las enfermedades y otros fenómenos que les impiden ser productivas. Esto explica que se deban analizar múltiples factores que afectan a esta problemática, además de los factores económicos tradicionalmente empleados. Asimismo, y como queda reflejado en los principales instrumentos internacionales en materia de desarrollo sostenible, la erradicación de la pobreza solo será posible si se tienen en cuenta los aspectos sociales, económicos y medioambientales del desarrollo (Díaz Barrado, 2018:34). Por ello resulta imprescindible adoptar una perspectiva que contemple precisamente estas dimensiones: Social: como seres humanos, nuestro bienestar está vinculado al de las demás personas. El aumento de la desigualdad es perjudicial para todos, ya que perjudica el crecimiento económico y repercute en la cohesión social, ocasionando situaciones de inestabilidad y conflicto; Política: mediante la utilización de políticas publicas inclusivas y basadas en el respeto de los derechos

\footnotetext{
${ }^{7}$ Mayor información ver en: https://www.un.org/sustainabledevelopment/es/poverty/
} 
humanos, los gobiernos pueden ayudar a construir un entorno propicio para generar empleo productivo y oportunidades de empleo para los pobres y los marginados; Medioambiental: el cambio climático, acompañado de fenómenos, tales como el "Niño" en algunas regiones del mundo y la sequía en otros, ha provocado que los trabajadores agrícolas busquen nuevas oportunidades de desarrollo en la ciudad. Dado que no siempre lo consiguen, éstos terminan engrosando los índices de pobreza en las periferias de las grandes ciudades. Por lo tanto, el cuidado del medio ambiente influye de manera directa no sólo en la producción de alimentos del campo y de primera necesidad, sino también en el mantenimiento de viviendas dignas que sean resistentes a las contingencias ambientales y fenómenos naturales, así como en el cuidado de la salud al contar con agua potable y de calidad y de un medio ambiente libre de polución, por lo que es imprescindible diseñar estrategias medioambientales basadas en los derechos humanos, que contribuyan a la disminución de la pobreza y el logro de los ODS.

En cualquier caso, para conceptualizar las múltiples dimensiones de la pobreza, el economista A. B. Atkinson en su informe a la Comisión para la medición de la pobreza mundial ha recomendado al Banco Mundial la incorporación de nuevas formas de medir y conceptualizar la pobreza: -Reconocimiento de personas que puedan definirse como pobres respecto a la sociedad en la que viven, incluso teniendo ingresos superiores a los 1.90 dólares diarios; “Incluir elementos básicos de bienestar, como saneamiento y servicios básicos de salud; - Ir más allá de la medición por hogares y medir la pobreza a niveles individuales; y, - La pobreza no solo entraña cuestiones económicas o de ingresos, sino que afecta a otros aspectos de la vida, tan esenciales para su bienestar, como la educación, la salud, el acceso a los servicios básicos y la seguridad" (Banco Mundial, 2018:6).

\section{Proporción de personas que viven en pobreza multidimensional, circa 2013}

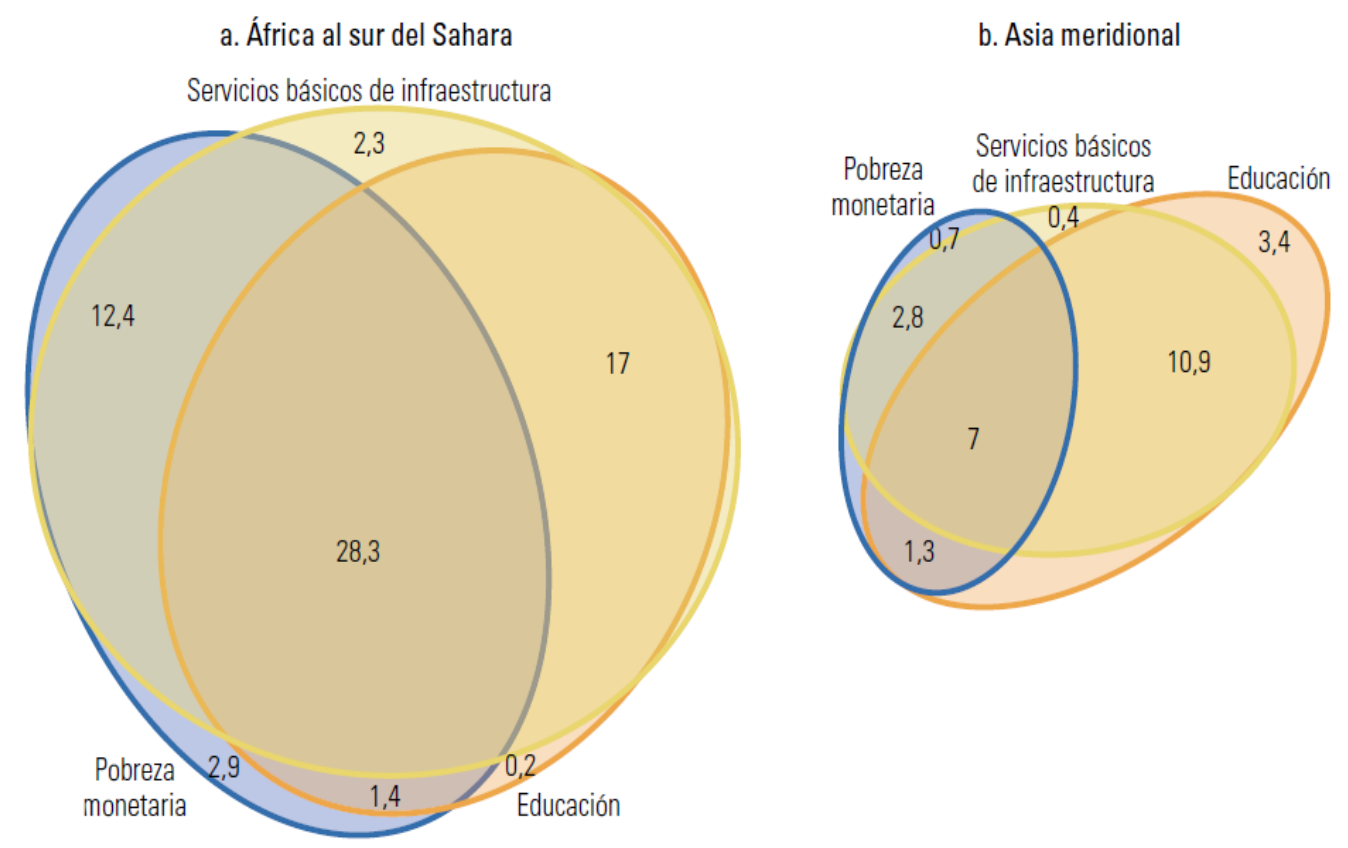

Fuente: Estimaciones basadas en las encuestas armonizadas de hogares de 119 economías (circa 2013), Base de Datos sobre Seguimiento Mundial, Grupo de Soluciones Mundiales sobre Medición del Bienestar y Fortalecimiento de la Capacidad, Departamento de Prácticas Mundiales de Reducción de la Pobreza y Promoción de la Equidad, Banco Mundial, Washington, DC.

Nota: El diagrama muestra la proporción de la población que vive en la pobreza multidimensional, y las dimensiones en las que sufre carencias. El tamaño de los óvalos está a escala para representar las respectivas proporciones en cada región. Por ejemplo, las cifras consignadas en el óvalo azul para África al sur del Sahara suman $44,9 \%$, que es el índice de recuento de la pobreza monetaria. La suma de todas las cifras consignadas para África al sur del Sahara da por resultado $64,3 \%$, que es la proporción de personas que sufren carencias en múltiples dimensiones. 
Como se puede observar en el gráfico anterior, las múltiples dimensiones que afectan la pobreza, y que son casi tan importantes como la monetaria, son la educación, los servicios básicos de infraestructura, la salud o la vivienda, principalmente. Como bien lo ha señalado Jim Yong Kim, Presidente del Grupo Banco Mundial "a menos que podamos acelerar nuevamente el crecimiento mundial y reducir la desigualdad, corremos el riesgo de no alcanzar la meta de poner fin a la pobreza extrema para 2030, establecida por nuestra institución. El mensaje es claro: para poner fin a la pobreza, debemos lograr que el crecimiento beneficie a los más pobres, y una de las medidas más eficaces en ese sentido es reducir el elevado nivel de desigualdad, en particular en los países donde residen muchas personas pobres"8.

\section{Los derechos humanos en las estrategias de combate a la pobreza: Agenda 2030}

"Si rescatamos un alma en medio de la pobreza, estamos defendiendo los derechos humanos. Y si fracasamos en esta misión, estamos fracasando con los derechos humanos"

- Kofi Annan, Ex Secretario General de las Naciones Unidas

(PNUD, 2007:6)

Las personas que viven en situación de pobreza son, en la mayoría de los casos, excluidas y desatendidas tanto por los gobiernos como por diversos sectores de la sociedad, produciéndose la violación de un gran número de derechos. Es por ello que resulta imposible desvincular estos dos conceptos, dada la interdependencia que existe entre ellos. En verdad, no se puede pensar en la pobreza sin hacer referencia a los derechos humanos, ya que la pobreza es tanto una causa como una consecuencia de la falta de la aplicación y cumplimiento de las normas en materia de derechos humanos. En ese sentido, el Programa de Acción y la Declaración de Viena de $1993^{9}$, constituyeron la primera iniciativa tendente a vincular estos dos conceptos enfatizando en que: "la pobreza extrema y la exclusión social constituyen un atentado contra la dignidad humana y que urge tomar medidas para comprender mejor la pobreza extrema y sus causas, en particular las relacionadas con el problema del desarrollo, a fin de promover los derechos humanos de los más pobres" (Asamblea General de las Naciones Unidas, 1993:8).

Posteriormente, la vinculación de estos dos conceptos se pudo advertir tanto en el Informe de Desarrollo Humano 2000, que consideraba la pobreza como una violación de la libertad y que solo su eliminación debería abordarse como un derecho básico y un derecho humano; como en la Nota Práctica del PNUD sobre la Reducción de la Pobreza y los Derechos Humanos (2003), que lo subrayaba en la frase de apertura pronunciada por Mary Robinson (Alto Comisionado para los Derechos Humanos, 1997-2002), al afirmar que "cuando me preguntan cuál es la violación del derecho humano más seria en el mundo hoy, mi respuesta es consistente: la pobreza extrema" (PNUD, 2000:8).

Ahora bien, en septiembre de 2012, el Consejo de Derechos Humanos aprobó por consenso la Resolución 21/11 que contiene los "Principios Rectores sobre la Extrema Pobreza y los Derechos Humanos". Estos Principios se centran básicamente en la promoción de los derechos humanos de las personas en situación de pobreza para que los gobiernos aseguren, mediante la implementación de políticas públicas, estrategias de erradicación de la pobreza, para que se respeten y defiendan sus derechos y se tomen en cuenta y eliminen los obstáculos sociales, culturales, económicos y estructurales que enfrentan las personas pobres para gozar de sus derechos. En cualquier caso, "su principal objetivo es orientar sobre la aplicación de las

\footnotetext{
${ }^{8}$ Véase en: https://www.bancomundial.org/es/news/press-release/2016/10/02/tackling-inequality-vital-toend-extreme-poverty-by-2030.

${ }^{9}$ Adoptada en la Conferencia Mundial sobre los Derechos Humanos, en Viena en 1993
} 
normas de derechos humanos en las estrategias de combate contra la pobreza" (Alto Comisionado de Naciones Unidas para los Derechos Humanos, 2012:4).

En este sentido, debe ser concebido como un instrumento útil para diseñar y aplicar políticas de reducción y erradicación de la pobreza. Cabría resaltar, como aspectos centrales de estos Principios Rectores, los siguientes: - Dignidad, universalidad, indivisibilidad, relación mutua e interdependencia de todos los derechos; - Disfrute en condiciones de igualdad de todos los derechos humanos por las personas que viven en la pobreza extrema; - Igualdad entre hombres y mujeres; - Derechos del niño; - Capacidad de actuación y autonomía de las personas que viven en la pobreza extrema; - Participación y empoderamiento; - Transparencia y acceso a la información; y - Rendición de cuentas (A/RES/21/11, 2012:5-13).

La premisa de erradicar la pobreza extrema, según los Principios Rectores, no solo es un deber moral sino también una obligación jurídica en el marco de la normativa internacional de los derechos humanos vigentes (A/RES/21/11, 2012:3). Sin embargo, los obstáculos con los que se enfrentan las personas en situación de pobreza para ejercer sus derechos son enormes, los cuales además de ser de índole económica, son también políticos, culturales y sociales. Por esto, una de las mejores estrategias para combatir la pobreza extrema es la incorporación del Enfoque Basado en los Derechos Humanos (EBDH) en las políticas públicas, ya que el EBDH proporciona un marco estratégico para erradicar la pobreza extrema a largo plazo mediante el reconocimiento a las personas que la padecen como titulares de derechos y agentes de cambio. Este Enfoque promueve el respeto de la dignidad y autonomía de las personas en situación de pobreza, al tiempo que las empodera para que puedan participar de manera provechosa y efectiva en la vida pública. No obstante, para reforzar los objetivos contenidos en el EBDH, se diseñó un Entendimiento Común que expresa que todas las agencias de Naciones Unidas deberán adherir a sus programas los siguientes lineamientos de dicho Entendimiento (PNUD, 2007:14):

- Todos los programas de cooperación al desarrollo y asistencia técnica deben fomentar la realización de los derechos humanos tal como lo establece la Declaración Universal de Derechos Humanos y otros instrumentos internacionales de derechos humanos.

- Los estándares contenidos tanto en la Declaración Universal de Derechos Humanos como en otros instrumentos internacionales, guiarán toda la programación y cooperación al desarrollo en todos los sectores y fases del proceso de programación.

- La cooperación al desarrollo contribuye al desarrollo de las capacidades de los titulares de deberes para cumplir con sus obligaciones y de las capacidades de los titulares de derechos para reclamar por sus derechos.

Las personas que suelen tener mayores dificultades para obtener acceso a ingresos, bienes y servicios son, en la mayoría de los casos, las mujeres, los niños, las personas con discapacidad, las personas mayores, los grupos indígenas, los migrantes y los desplazados. En otras palabras, personas que se podrían incluir dentro de los denominados "grupos vulnerables". En esta línea, destaca también que la estrategia utilizada por los países con altas tasas de población en situción de pobreza es la focalización de sus políticas sociales hacia los más pobres de entre los pobres (PNUD, 2007:14). En esencia, el EBDH subraya que lo más importante es dar prioridad a los grupos más vulnerables pero sin olvidar al resto de la población que también se pudiera encontrar en situación de vulnerabilidad, y siempre garantizando un nivel minimo básico en cada uno de los derechos de toda la población para mantener un nivel de bienestar más generalizado. Con esta estrategia de focalización se genera una dinámica de discriminación hacia el resto de la población vulnerable que suele ser considerada dentro de los programas de ayuda. Por lo que desde el enfoque de derechos, la inclusión activa de la población en general y, sobre todo, de la población más vulnerable es una 
de los aspectos prioritarios para disminuir y combatir los casos de discriminación (Verdiales, 2018).

Sin embargo, tanto el EBDH como los "Principios Rectores sobre Extrema Pobreza y Derechos Humanos" así como los Objetivos y Metas plasmados en la Agenda 2030 son solo, en la actualidad, un marco de principios y procedimientos de carácter recomendatorio para los Estados, los cuales pueden, en caso de así decidirlo, implementarlos en la formulación de sus políticas públicas. Es por ello que las estrategias de erradicación de la pobreza se ven muchas veces afectadas por los intereses de los Estados, quienes en algunas ocasiones pueden alejarse de las recomendaciones internacionales sobre igualdad, equidad y desarrollo sostenible, poniendo en riesgo el bienestar de millones de personas. En cualquier caso, como lo indica Cástor Díaz Barrado, cabría afirmar que, por ahora, la práctica internacional reconoce que el fin de la pobreza se puede abordar desde el enfoque de los derechos humanos, indicando para ello que los tres principios más destacados en el orden internacional para erradicar la pobreza son precisamente aquellos que se han señalado, es decir (Díaz Barrado, 2018:32):

- El principio de la Cooperación Internacional: este principio, fundamentado en los artículos 55 y 56 de la Carta de las Naciones Unidas, es imprescindible en la erradicación de la pobreza ya que mediante la cooperación internacional se ayuda y asiste a los grupos más vulnerables de la sociedad internacional. Aunque sus objetivos son bastante genéricos y no constituyen una obligación como tal para los Estados, su práctica y desarrollo son de vital importancia para el logro de los Objetivos de desarrollo, basados en la solidaridad y en la igualdad social, en beneficio de toda la sociedad internacional.

- El principio del Desarrollo Sostenible: los ODS, y anteriormente los ODM, constituyen y han constituido un marco de referencia inexcusable para la promoción de un desarrollo sostenible, incluyente e igualitario en la comunidad internacional. Por lo tanto, son el referente internacional para la promoción de los derechos humanos mediante mecanismos de desarrollo humano sostenible.

- El principio de la Protección Internacional de los Derechos Humanos: para lograr la erradicación de la pobreza en el planeta es imprescindible vincular la pobreza con los derechos humanos, ya que esta vinculación hará posible el establecimiento de marcos jurídicos y de protección de los grupos más vulnerables que a su vez mejoraran sus condiciones de vida. El reconocimiento de derechos básicos como acceso a una vivienda digna, a una educación de calidad, a servicios médicos, a un trabajo decente, construirán el pleno desarrollo de la persona, generando a su vez desarrollo a su alrededor y combatiendo de forma sistémica los círculos de la pobreza. En este sentido, la aplicación de los "Principios Rectores sobre la Extrema Pobreza y los Derechos Humanos", así como las estrategias contenidas en el EBDH en las políticas públicas de los Estados con mayores índices de pobreza, contribuirán en gran medida en la mejora de las condiciones de vida de los grupos más vulnerables y, por ende, a la erradicación de la pobreza en el mundo.

\section{Conclusiones}

La pobreza es un fenómeno multidimensional ya que no solo abarca las cuestiones económicas, sino que afecta, también, la integridad y dignidad de las personas al ver limitada o restringida su participación en cuestiones sociales, políticas o culturales. Como es sabido, para lograr el desarrollo humano que permita la construcción de una sociedad internacional justa, solidaria y equitativa, es imprescindible el combate y la erradicación de la pobreza y, en este sentido, los derechos humanos juegan un papel fundamental. Puesto que los derechos humanos son aquellas garantías individuales que emanan de los ordenamientos internacionales, regionales o nacionales que interfieren en la promoción y respeto de los derechos y las libertades humanas en los ámbitos sociales, económicos, culturales, políticos y civiles, velando por el respeto de la 
dignidad humana resulta, por lo tanto, evidente la vinculación de ambos conceptos al existir un alto grado de interdependencia.

La comunidad internacional ha realizado grandes esfuerzos para combatir y erradicar la pobreza existente en el mundo. Si bien estos esfuerzos comenzaron tras la Segunda Guerra Mundial, éstos se vieron opacados, no obstante, durante el periodo de la Guerra Fría. Por lo que no fue sino hasta el decenio de los noventa del siglo XX, cuando se dio un verdadero giro hacia el combate de la pobreza haciendo referencia a los principios en materia de derechos humanos. Esta vinculación fue haciéndose cada vez más sólida y notoria por su inclusión en la Declaración de Viena (1993) y posteriormente en el Informe de Desarrollo Humano (2000) así como en la Nota Práctica del PNUD sobre la Reducción de Pobreza y los Derechos Humanos (2003).

Un momento clave para el desarrollo humano sostenible fue la celebración de la Cumbre del Milenio (2000) de la cual se derivó la Declaración del Milenio, la cual contenía 8 Objetivos generales para trabajar en la construcción de un mundo más igualitario y equitativo. Los ODM supusieron un gran avance en materia de reducción de la pobreza y la pobreza extrema. Sin embargo, esto no fue suficiente para poder erradicar los altos niveles de pobreza existentes en el planeta pese a que los avances producidos fueron bastante importantes. En realidad, no se logró integrar a esos millones de personas que sufren día a día no solo carencias físicas y económicas sino también sociales y culturales. Por lo que, nuevamente, la comunidad internacional quiso valorar dichos resultados y proponer una nueva serie de Objetivos que dieran continuidad a las estrategias implementadas en los ODM y que incluyeran aquellas acciones que demanda la actual sociedad internacional. En tal sentido, el Objetivo sobre erradicación de la pobreza se volvió a incluir, como el primero de todos, en los ODS en un nuevo periodo de cumplimiento de 15 años tanto en los países en desarrollo como en los países desarrollados, evitando de esta forma cualquier tipo de disparidad entre las regiones del mundo.

Adicional a estos instrumentos internacionales de desarrollo, se adoptaron otra serie de principios y enfoques que contribuyeran a la erradicación de la pobreza, así como a la promoción de los derechos humanos, como son los "Principios Rectores sobre Extrema Pobreza y Derechos Humanos" y el Enfoque basado en los Derechos Humanos, los cuales trabajan en la construcción de estrategias para la erradicación de la pobreza con base en principios básicos en materia de derechos humanos. Esta constituye una de las formas más sólidas para la erradicación de la pobreza y por ende, para la construcción del desarrollo humano sostenible que tanto demanda la sociedad internacional. No obstante, como hemos hecho mención, los instrumentos internacionales destinados a erradicar la pobreza tienen en la actualidad, carácter recomendatorio para los Estados, por lo que se requiere de un verdadero compromiso por parte de los líderes políticos para garantizar la aplicación de la normativa internacional en materia de erradicación de la pobreza desde la promoción y respeto de los derechos humanos.

En definitiva, resulta imprescindible la aplicación de estrategias basadas en el refuerzo y promoción de los derechos humanos, tales como el EBDH. Su incorporación resulta importante pero, también, necesaria si se quiere erradicar la pobreza que tanto afecta a millones de personas alrededor del mundo. Puesto que, por primera vez en la historia de la humanidad, las condiciones económicas lo permiten no tendríamos excusas para no poder hacerlo.

\section{Bibliografía}

ALTO COMISIONADO DE NACIONES UNIDAS PARA LOS DERECHOS HUMANOS (2012). Los principios rectores sobre la Extrema Pobreza y los Derechos Humanos, Resolución 21/11, Ginebra, Suiza

ASAMBLEA GENERAL DE NACIONES UNIDAS (1993). Conferencia Mundial de Derechos Humanos, Viena, Austria 
ASAMBLEA GENERAL DE NACIONES UNIDAS (2000). Declaración del Milenio, A/RES/55/2, 2000, Nueva York, NY

AS AMBLEA GENERAL DE NACIONES UNIDAS (2009). Segundo Decenio de las Naciones Unidas para la Erradicación de la Pobreza (2008 2017), A/RES/63/230, Nueva York, NY

ASAMBLEA GENERAL DE NACIONES UNIDAS (2010). Actividades del Segundo Decenio de las Naciones Unidas para la Erradicación de la Pobreza (2008-2017), A/65/230, Nueva York, NY

AS AMBLEA GENERAL DE NACIONES UNIDAS (2015). Memoria del Secretario General sobre la labor de la Organización, A/RES/70/1, Nueva York, NY

BANCO MUNDIAL/Banco Internacional de Reconstrucción y Fomento (2018). La pobreza y la prosperidad compartida 2018: Armando el rompecabezas de la pobreza, $1818 \mathrm{H}$ Street NW, Washington, DC, 20433

COMITÉ DE DERECHOS ECONÓMICOS, SOCIALES Y CULTURALES (2002). Informe sobre los períodos de sesiones vigésimo quinto, vigésimo sexto y vigésimo séptimo (23 de abril a 11 de mayo de 2001, 13 a 31 de agosto de 2001 y 12 a 30 de noviembre de 2001), E/2002/22, E/C.12/2001/17, p. 208

DÍAZ BARRADO, C. M. (2018). La erradicación de la pobreza y derechos humanos: un laberinto sin salida, Revista Derechos y Libertades, No 38, Época II, pp. 17-52, ISSN: 11330937, DOI: 10.14679/1055

NACIONES UNIDAS, CONSEJO ECONÓMICO Y SOCIAL (2018). Observaciones formuladas en ausencia del informe inicial del Congo, aprobadas por el Comité en su $49^{\circ}$ período de sesiones (12 a 30 de noviembre de 2012), E/C.12/COG/CO/1

NACIONES UNIDAS (2015). Objetivos de Desarrollo del Milenio: Informe de 2015, Nueva York, NY., págs. 72. https://www.undp.org/content/undp/es/home/librarypage/mdg/themillennium-development-goals-report-2015.html

OFICINA INTERNACIONAL DEL TRABAJO, La Iniciativa para poner fin a la pobreza: La OIT y la Agenda 2030, Conferencia Internacional del Trabajo 105ª reunión, 2016, Ginebra, p. 1

PNUD (2007). Operacionalización de los enfoques basado en los derechos humanos para la reducción de la pobreza. Informe provisorio sobre un proyecto piloto, Nueva York, págs. 43

SUSTAINABLE DEVELOPMENT SOLUTIONS NETWORK (2018). SDG Index and Dashboards Report 2018. Global Responsibilities implementing the goals, Bertelsmann Stiftung and Sustainable Development Solutions Network

VERDIALES LÓPEZ, D. M. (2018). La importancia del Enfoque de Derechos Humanos en los Objetivos de Desarrollo Sostenible, en DÍAZ BARRADO, C. M. y FERNÁNDEZ LIESA, C. R. (dirs.), VERDIALES LÓPEZ, D. M. (coord.). Objetivos de desarrollo sostenible y Derechos Humanos: paz, justicia e instituciones sólidas / Derechos Humanos y empresas, Editorial: Instituto de Estudios Internacionales y Europeos Francisco de Vitoria de la Universidad Carlos III de Madrid, pp. 75 - 90 